\title{
Key Performance Indicators for Evaluating Corporate Wikis for Knowledge Management
}

\author{
Markus Michael Mützel \\ Evonik Industries AG, Germany \\ markus.muetzel@evonik.com
}

\begin{abstract}
This paper presents a methodology for assessing and improving the quality of information provided by corporate wikis. Regarding the assessment, we present two KPIs for measuring relative demand and relative usefulness of wiki articles, including corresponding processes and data model. In regard to improving quality, we use the KPIs to classify the articles. For this classification, we introduce four categories and discuss possible actions for reducing information overload and increasing the visibility of articles. To prove our methodology, we analyze an existing corporate wiki of a large European enterprise in the chemical industry. Its articles are used to demonstrate how the proposed KPIs can contribute to knowledge management by improving the information quality.
\end{abstract}

\section{Introduction}

From the enterprises' point of view, knowledge has always had an influence on their competitiveness. In course of time, enterprises realized that knowledge became more and more a critical success factor in addition to the classical resources land, labor, and capital [1], [2]. Nowadays, efficient means for production and distribution of information are available but the meaningful reduction and gathering of relevant information is still challenging [3]. The advent of knowledge management and according information systems was the result of those challenges. Only an efficient knowledge management can help to improve internal processes and products, in order to generate a competitive advantage [4], [5]. Web 2.0 technologies, e.g., wikis, provide an obvious foundation for efficient knowledge management and have been widely used for that purpose [6].

On the one hand, a wiki can support decentralized information gathering and central distribution, but on the other hand it can increase the quantity of available information. This effect can lead to information overload. [7] discusses the causes and the negative consequences of information overload, e.g., wrong decisions or inefficient work. This paper provides a solution to the problem of inefficient usage of wikis in enterprises. In other words, it delivers the answer

\author{
Omid Tafreschi \\ Hochschule Darmstadt \\ University of Applied Sciences, Germany \\ omid.tafreschi@h-da.de
}

to the question: How to evaluate and improve a wiki as an efficient tool for knowledge management?

For this purpose, the paper delivers an instrument to monitor the development of wikis in order to minimize information overload and counteract undesirable developments. Key Performance Indicators (KPIs) [8] can provide an instrument for evaluating a wiki as a tool for efficient knowledge management. This paper presents two KPIs for that purpose.

The paper is organized as follows. After the introduction, Section 2 discusses wikis as a tool for knowledge management, focusing on information quality and its interdependencies with information supply, information need and information demand. Section 3 presents the processes for calculating the KPIs and the underlying data model. In order to cluster and interpret the KPIs, Section 4 proposes a classification matrix. Section 5 shows an example of the usage of the KPIs in an existing corporate wiki. Section 6 discusses the related work and Section 7 provides the conclusion and future work.

\section{Corporate Wikis for Efficient Knowledge Management in Enterprises}

In this section the concept of a wiki and its potential for knowledge management within enterprises is explained. Then an approach for assessing information quality and an approach for evaluating wikis, considering the demand and supply of information within enterprises, is discussed.

\subsection{Wikis within Enterprises}

Web 2.0 is not a single technology or philosophy, it is an accumulation from different philosophies, technologies and applications focusing on the possibility to contribute information in a cooperative and interactive manner. This idea led to the advent of different applications such as wikis, blogs, or social networks [9].

A wiki is a web application, which doesn't require a local software installation. It consists of several articles 
linked together. The concept of wikis doesn't only include the search for information by users in a central database, but also enables them to create and edit information. This means every user can contribute information and link it to existing information in order to create a collaborative information platform. According to [4], this linkage creates new knowledge.

[10] provides the SECI model for describing the process of knowledge creation and utilization. According to that model, the creation of new knowledge is a spiral, which creates knowledge through the interaction between explicit and tacit knowledge. This transformation of individual tacit knowledge in organizational explicit knowledge, is defined by four process steps of knowledge conversion, i.e., socialization, externalization, combination, and internalization [11]. Wikis can spur this conversion, e.g., the short time gap between the documentation and communication of knowledge or the possibility to link related information. Hence, wikis provide an obvious foundation for efficient knowledge management in companies. In this case wikis are called corporate wikis [12]. [13] presents a survey discussing various aspects of the wiki usage within enterprises and analyzes its potential for knowledge management.

The distinction between open wikis, e.g., Wikipedia, and corporate wikis is an important aspect for our further analysis. The users of corporate wikis are well known and differentiated with special authorizations. The positive influence of known users in contrast to anonymous users, e.g., disruptive behavior of anonymous users, is researched by [14]. In addition, the purpose of corporate wikis can differ from open wikis, since corporate wikis aim at creating business value. However, all kinds of wikis have to face the issue of information overload [15]. An information overload can affect the performance of an information requester negatively, so the value of a corporate wiki is reduced [7]. Value loss is a relevant problem of corporate wikis and the knowledge management. This paper addresses this problem by providing measures for the reduction of the value loss caused by information overload. For this purpose, we analyze an accepted approach for the definition of information quality and a related framework for describing the relations between information demand, supply, and need in the following sections.

\subsection{Information Quality}

There is a body of literature about information and knowledge quality [5], [16], [17]. All studies define similar dimensions for assessing quality and group them into categories. We use the approved framework provided by [17] for analyzing the quality of a corporate wiki. It defines the usefulness and usability of information as significant factors for assessing information quality and introduces four categories with different information quality dimensions depicted in Table 1.

Table 1. Information quality categories and dimensions [17]

\begin{tabular}{|c|c|}
\hline $\begin{array}{c}\text { Information Quality } \\
\text { Category }\end{array}$ & $\begin{array}{c}\text { Information Quality } \\
\text { Dimensions }\end{array}$ \\
\hline Intrinsic & Accuracy, Objectivity, \\
& Believability, Reputation \\
\hline Accessibility & Accessibility, Access security \\
\hline Contextual & $\begin{array}{c}\text { Relevancy, Value-Added, Timeliness, } \\
\text { Completeness, Amount of data }\end{array}$ \\
\hline Representational & $\begin{array}{c}\text { Interpretability, Ease of understanding, } \\
\text { Concise representation, } \\
\end{array}$ \\
& Consistent representation \\
\hline
\end{tabular}

The information quality dimensions are interdependent and so they can cause information quality problems which are discussed by [17] with help of patterns. In case of wikis, this interdependency can be found when multiple articles with the same information are created. This can reduce the believability and impede the users to update all relevant articles with the newest information, which endanger the accuracy of information. The consequence of questionable believability and accuracy can lead to a low reputation. As a consequence, the information is not used by the information requester. This case depicts the information requester perspective emphasized by [17]. The information has to fit for use by the information requester.

This perspective of the information requester regarding the information quality is helpful for the analysis of wikis and the information quality of its articles. Useful articles face a high demand by information requesters. Furthermore, they can also edit articles with information quality problems to improve the value for other users. The usefulness of information can be influenced by the information overload. [7] shows the negative impacts, e.g. inconsistent information or the lack of critical evaluation of information. In these cases, information does not fit for being used by the information requester.

\subsection{Information Demand and Supply}

According to [17], the perspective of the users have to be analyzed regarding information quality. This perspective includes the needs of the users and their behavior. These can be analyzed by the framework provided by [18], which explain the relationship between information need, information demand, and information supply. This framework is depicted in Figure 1.

The subjective information need is the estimation of a specific employee regarding the information required for his tasks. In other words, the subjective information need reflects an individual perspective. This can differ from the objective information need, i.e., the information actually required for the tasks. The objective information need is independent from a specific employee. The information 


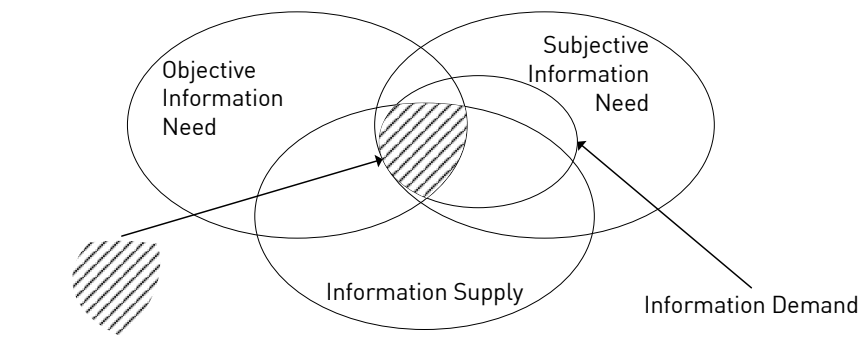

Level of Information

Figure 1. Information need vs. information demand vs. information supply [18]

demand is a subset of the subjective information need and represents the actions of that employee, e.g., searching in a corporate wiki. The subjective information need and the objective information need can overlap, but information demand is always a subset of the subjective information need.

Information supply is the total amount of available information, e.g., provided by all articles of a corporate wiki. The level of information is the intersection of objective information need, information demand and information supply. This intersection represents the business relevant knowledge and thus has to be maximized. Two steps are required for that purpose. First, the amount of information supply can be modified, e.g., the enrichment of relevant information in an article, to increase its relative overlap with objective information need. Second, the content of the information supply can be adjusted, e.g., revising the title of an article, to emphasis its relevance. Both approaches are related to the information supply which has different intersections with the information need and information demand.

For a corporate wiki, its articles represent the information supply. Considering a single wiki article, either it is not demanded or it is demanded. The demand is determined by article views which can be direct, i.e., by opening the article, or indirect, i.e., the article is shown as a search result. Articles without views represent a subset of information supply without corresponding demand. Figure 2 depicts the two subsets of information supply.

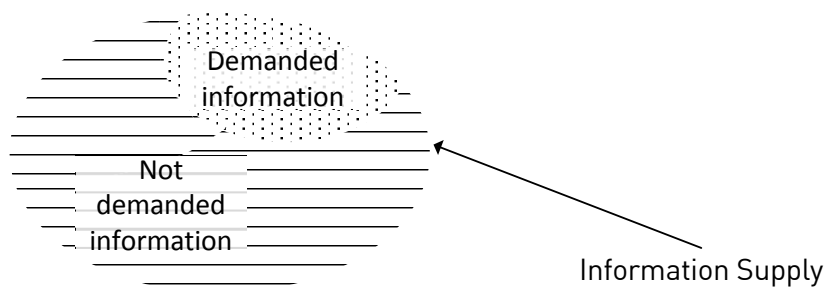

Figure 2. Demanded vs. not demanded information

Since information demand does not necessarily represent the usefulness of information, we further diversify the information supply with the help of information need. Therefore we differentiate between objective and subjective information need as proposed by [18]. This differentiation is shown in Figure 3 in which a wiki article is assigned to one of the following four sets:

Set I contains articles which are not objectively needed and are not demanded.

Set II contains articles which are not objectively needed but are demanded.

Set III contains the valuable subset of articles which are objectively needed and are demanded.

Set IV contains articles which are objectively needed but are not demand.

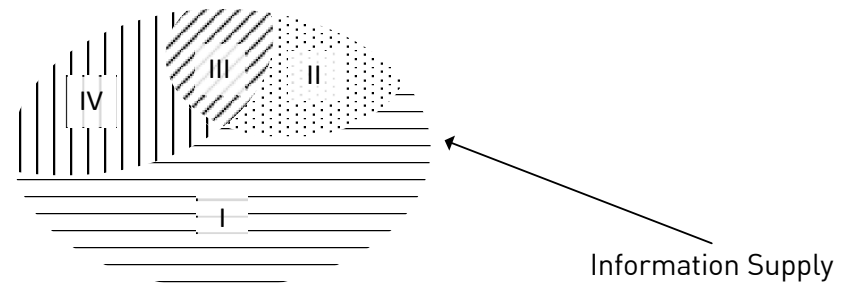

Figure 3. Categories of information supply

All articles of a corporate wiki can be assigned to the aforementioned four sets. In order to improve the business value of a corporate wiki those four sets have to be optimized. For that purpose we present two KPIs in the following section.

\section{Key Performance Indicators}

In this section we first explain our approach for calculating KPIs for assessing the quality of wiki articles using the frameworks described in Sections 2.2 and 2.3. Then we define the necessary data model and two processes for calculating the KPIs.

\subsection{Approach}

In Section 2.2 we discussed the definition of information quality and the relevance of usefulness provided by [17]. In Section 2.3 we analyzed the framework proposed by [18] and especially the differentiation between objective and subjective need. Combining these two results, we introduced four different information sets in Figure 3. The intersection of the information supply and the objective information need, i.e., sets III and IV, contains the valuable subsets. These are actually relevant and useful for the enterprise. Thus, it is important to increase this intersection by supplying corresponding articles. The difference between the two subsets is the demand. While set III is demanded, set IV is disregarded by the users. There is no objective need 
for the articles in sets I and II. Therefore, the usefulness of these articles is not given, although set II is demanded by the users.

As stated above, information demand and the information usefulness have to be quantified with help of KPIs in order to assess the information quality. This quantification requires input parameters. For the KPI reflecting information demand, we analyze the views of every article within a specific time frame, i.e., period of analysis. This time frame helps us to consider the evolution of a corporate wiki where all articles can be evaluated independently from their age.

For the KPI reflecting information usefulness of an article, we analyze different factors corresponding to the authors, i.e., the roles, the demand for articles, the cooperation between authors.

If an author can assess the relevance of information, he can create and edit useful articles. This step leads to the question, which author characteristics are helpful to write useful articles. These can be assessed by considering the role of an author, since it influences his contribution. E.g., a process owner usually knows most about the overall context, objectives and details of his process and is able to estimate the objective needs. In this case it is reasonable to assume that his articles are more useful than those created by authors having other roles. This assumption is supported by the study about expertise retrieval [19]. According to that study, the expertise of employees is an asset and its effective sharing is crucial from a company's point of view.

In addition to the role of an author, the demand for his articles can be used to assess the influence of an author. Furthermore, extending the focus considering the cooperation of different authors, i.e., number of edits by distinct authors, can be used to evaluate the quality of an article [20], [21].

\subsection{Data Model}

The data which is necessary for the calculation of the proposed KPIs is based on the class diagram depicted by Figure 4.

The class role is relevant for distinguishing the objective information need from the subjective one. Hence, it assigns the attribute ImpactFactor to a specific role. An object belonging to the class Author has his own Id, i.e., AuthorId, and a specific role, e.g., process owner, key user and employee. An object belonging to the class Article has an unique Id, i.e., ArticleId, and a Title. An object belonging to the class Edit reflects a write access done by a specific author to a specific article at a specific time. As a consequence, an object has three attributes, i.e., AuthorId, ArticleId, and Time. Objects belonging to the class View contain all read accesses, i.e., opening an article by a user at a specific time. These objects reflect the demand for the articles.

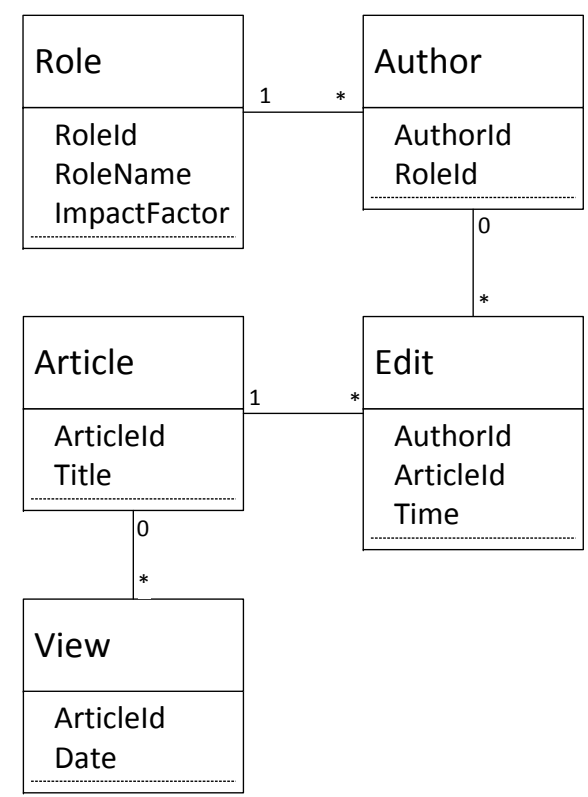

Figure 4. Data model

\subsection{Processes for Calculating the KPIs}

In the following, we propose the processes for calculating two KPIs for assessing information demand and information usefulness of articles contained in a corporate wiki. For this purpose, we use Business Process Model and Notation (BPMN) for defining the processes [22], since BPMN is the de-facto standard for process modeling [23].

3.3.1. KPI for Information Demand. The process shown in Figure 5 aims at assessing the information demand of all articles within a corporate wiki according to Figure 2. In other words, it assigns a specific wiki article to a KPI indicating its level of demand. We assume that this KPI is periodically calculated. Hence, the process starts at a specific time and considers a period of analysis which reflects a time frame in the past. This period is defined by a start time $(S T)$ and an end time $(E T)$. After the start the process performs following 6 activities:

1) This activity determines the creation dates of all articles contained in a corporate wiki. We refer to the creation date of an article as $C D$. For this, the activity retrieves the data contained in the database Write \& Read Accesses which stores the data according to the model shown in Figure 4.

2) This activity considers all articles. For each article the activity counts the number of its views $(\mathrm{NoV})$ during the period of analysis.

3) This activity considers all articles. For each article the number of views is adjusted if the creation date of the article was after the start time of the 


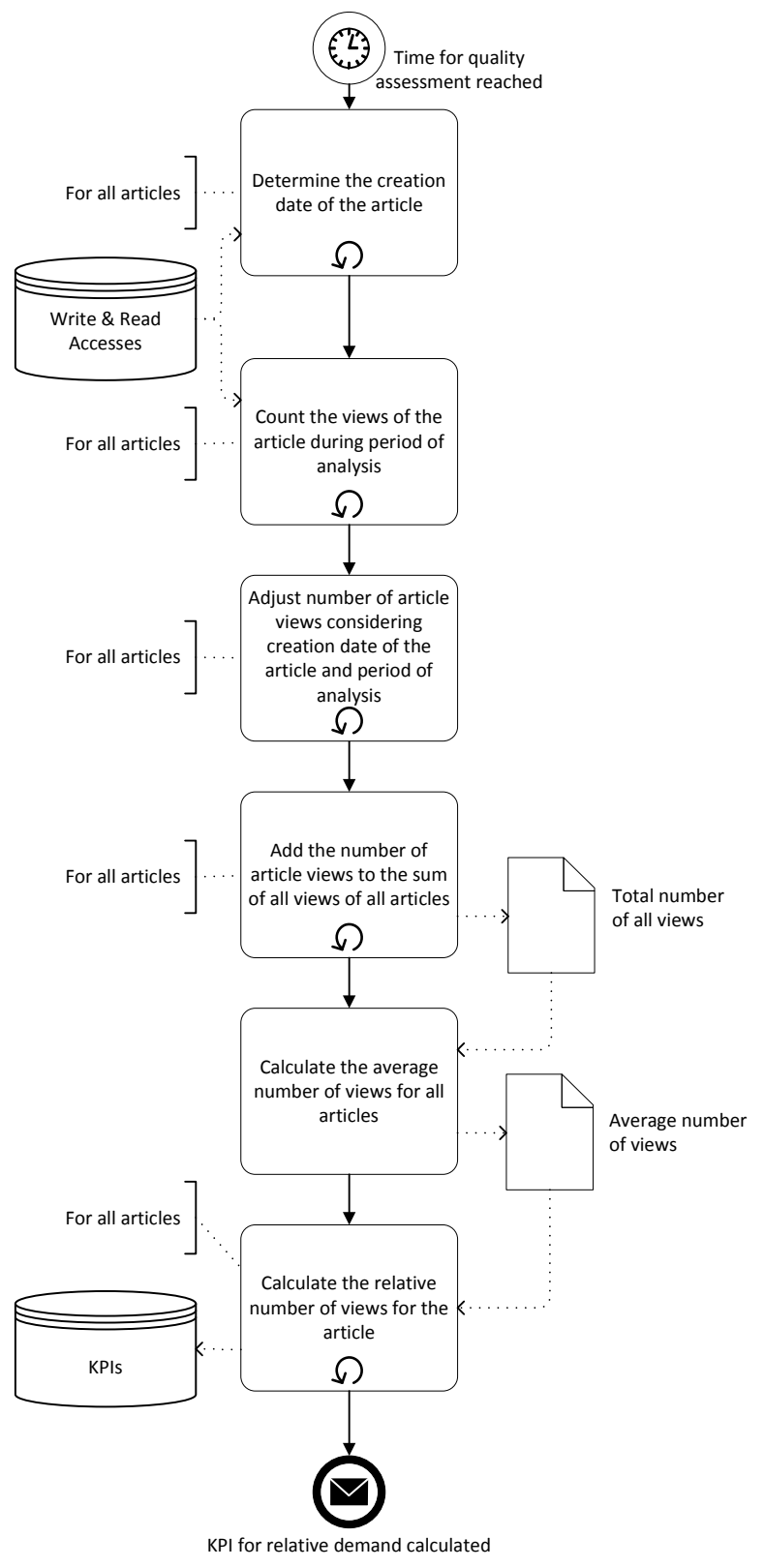

Figure 5. Process for calculating information demand

period of analysis. For this purpose, the following formula is used

$A N o V=N o V * \frac{E T-S T}{E T-C D}$

where $A N o V$ denotes the adjusted number of views of an article. This adjustment allows the evaluation of articles independently of their creation date.

4) This activity calculates the sum of all views of all articles within the period of analysis. The result is the total number of all views.
5) This activity calculates the average number of views of all articles with the help of the previously calculated total number of all views. The result of this calculation is the average number of views for all articles.

6) This activity calculates the relative number of views for a specific article considering the average number of views of all articles. The result is the KPI reflecting the information demand which is stored in the KPI database.

This previously described KPI is a relative number which allows the comparison of different articles of a corporate wiki.

3.3.2. KPI for Information Usefulness. The process depicted by Figure 6 aims at assessing the information usefulness of all articles within a corporate wiki according to Figure 3.

In other words, it assigns a KPI to a specific wiki article indicating its level of usefulness. It starts after the process for calculating information demand and performs the following 10 activities:

1) This activity assigns the authors to their articles.

2) This activity determines the total number of authors that contributed to an article.

3) This activity considers all authors of an article. Each author gets the number of article views divided by the number of its authors. The result is the partial number of all views of an article.

4) This activity sums the results of the previous step, i.e., each author gets an individual score, including all his partial numbers, indicating the demand for all this articles.

5) This activity adjusts the result of the previous step considering the roles of authors. For this, the impact factors indicating the knowledge level of authors are retrieved from the database Write \& Read Accesses. The calculation results are adjusted scores of all authors.

6) This activity considers all articles. Each article gets the sum of all adjusted scores of its authors, i.e., its partial usefulness.

7) This activity adjusts the partial usefulness of all articles considering the creator impact factor. This factor is an input parameter for the process. This approach helps to emphasize the relevance of an article considering its creator.

8) This activity determines the total usefulness of all articles by summing the results of the previous activity.

9) This activity calculates the average usefulness for all articles using the previous total usefulness. 


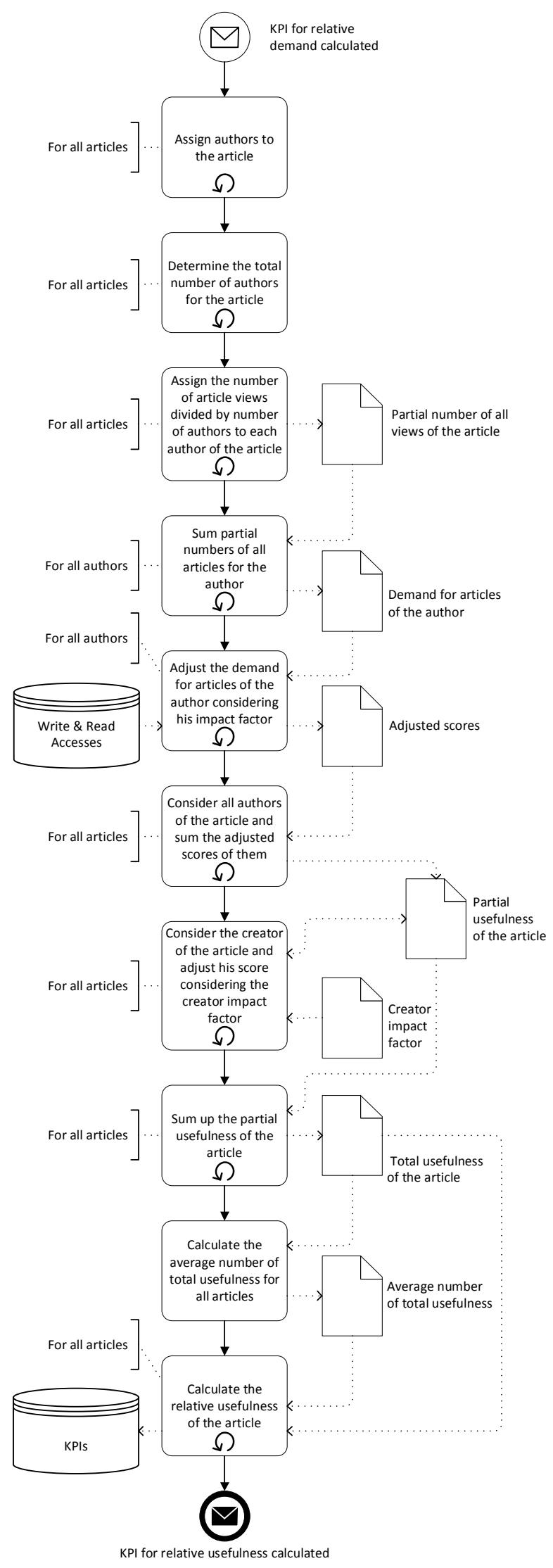

Figure 6. Process for calculating information usefulness
10) This activity considers all articles and calculates the relative usefulness of every single article using the total usefulness of that article and the average usefulness for all articles. The result is the KPI reflecting the usefulness of an article which is stored in the KPI database.

The previously described KPI is a relative number which allows the comparison of different articles of a corporate wiki

\section{Quality Classification of Wiki Articles}

The presented processes deliver two sets of KPIs for assessing the quality of articles contained in a corporate wiki. The next step is to classify those articles. For this purpose we use a matrix which is similar to the approved one proposed by [24] for describing the product life cycle. It has two dimensions, i.e., the market growth rate and the relative business position. With the help of that matrix, the products of an enterprise can be assigned in four categories, i.e., stars, cash cows, poor dogs, and question marks. The categories can help to classify the products and develop adequate strategies.

We use a similar concept to analyze the articles in an corporate wiki. We propose two dimensions, i.e., relative demand and relative usefulness. These dimensions lead to the following four categories:

\section{Inaccurate Information}

The articles in this category have low demand and low relative usefulness. These articles deserve low attention. In order to reduce information overload the deletion of these articles is conceivable. However, the reason for the bad quality could be due to inaccurate wording and can be improved by revising the content.

\section{Unreasonable Information}

Articles in this category have high demand but low usefulness. At first glance, the high demand is a benefit since it expresses the usage of the corporate wiki which in turn is a collaboration. However, measures for increasing the usefulness of those articles should be applied. These can include adding relevant information to those articles or linking to more useful articles belonging to the next category.

\section{Undisclosed Information}

This category contains articles that have high usefulness. This property is because of the involved authors. However, these articles face low demand. There can be three reasons for this phenomenon. First, the actual usefulness could be lower because of outdated content. In this case, removing the article from the corporate wiki would be reasonable. 
Second, the low demand is due to the limited visibility of the article, which can be the result of missing key terms or synonyms in the content. Additional links from articles of the previous category, i.e., unreasonable information, increase the visibility. In addition, mentioning most used search terms can help to overcome this shortcoming. Third, the set of employees demanding the content is small. This could be the case when experienced employees are using the corporate wiki while the target audience of an article are new employees. In this case, no action needs to be taken. If the three mentioned reasons don't fit then the awareness of the community about the article has to be analyzed and increased if necessary.

\section{Relevant Information}

Articles in this category are very important for the knowledge management. They have high demand and high usefulness. Therefore, these articles need a continuous quality review in order to keep the articles up to date.

\section{Evaluation}

We analyzed different existing corporate wikis by applying the proposed KPIs. In this section we present the evaluation of one of the analyzed corporate wikis which is used in a large European company in the chemical industry for the documentation of purchase-to-pay processes. Table 2 shows the data contained in the analyzed wiki corresponding to the model depicted by Figure 4 . In addition to the 54 authors, 61 employees use the corporate wiki to obtain information.

Table 2. Analyzed data

\begin{tabular}{|l|c|}
\hline Table & Number of Records \\
\hline Role & 4 \\
\hline Author & 54 \\
\hline Edit & 11675 \\
\hline Article & 467 \\
\hline View & 40919 \\
\hline
\end{tabular}

Before the data is used for the calculation of the KPIs, the impact factors and the period of analysis have to be defined. The latter was set to 365 days to cover a whole business year including different phases, e.g., year-end closing. With this period of analysis, we consider all articles even though they are only relevant for a specific time frame. Employees accessing the analyzed corporate wiki are assigned to four different roles, i.e., process owner, key user, user, and reader. The first three roles are used for authors.

Table 3. Role data

\begin{tabular}{|l|c|}
\hline Role & Impact Factor \\
\hline Process Owner & 2 \\
\hline Key User & 1.5 \\
\hline User & 1 \\
\hline
\end{tabular}

Table 3 shows the different roles and their impact factors reflecting the dependency of the objective information need from a specific role. Since numerous authors can contribute to the wiki, we set the creator impact factor to 1.5 to emphasize the influence of the creator on the objective information need.

We calculate the two KPIs presented in Section 3 using the data shown in Table 2. For the sake of clarity, Table 4 shows the results for 7 representative articles.

Table 4. Overview KPI

\begin{tabular}{|c|c|c|}
\hline ArticleId & Relative Demand & Relative Usefulness \\
\hline 1 & 2.33 & 1.76 \\
\hline 2 & 1.42 & 0.90 \\
\hline 3 & 0.40 & 0.79 \\
\hline 4 & 0.68 & 1.19 \\
\hline 5 & 0.15 & 0.30 \\
\hline 6 & 1.71 & 1.38 \\
\hline 7 & 0.31 & 0.68 \\
\hline
\end{tabular}

For assessing the quality of articles contained in the analyzed corporate wiki we use the matrix described in Section 4. Figure 7 depicts the result. In order to improve the overall quality of the corporate wiki we recommend to prioritize two actions. First, in order to reduce information overload articles 5 and 7 should be removed from the corporate wiki. Second, the visibility of article 4 should be improved by adding links to that article from article 2 or adding corresponding search terms which are often used by the employees to that article.

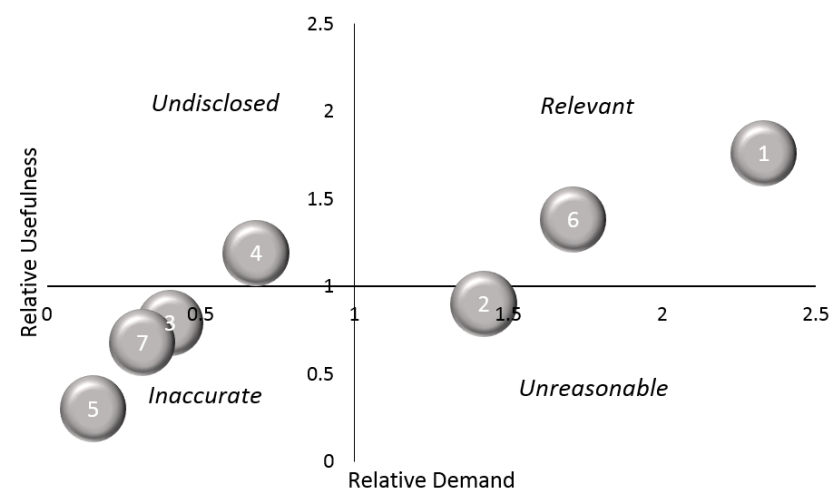

Figure 7. Matrix for assessing the KPIs

\section{Related Work}

Web 2.0 technologies have been evaluated for efficient knowledge management for companies. This evaluation was done from different perspectives regarding special contexts. [25] focuses on the content of an article and analyzes the interdependence of the quantity of words and the quality of the corresponding article. [20] assesses the influence of different unique authors on the quality of articles. Both 
are similar to our work since we use the information of authors to calculate the KPI for usefulness. However, we extend the approach by regarding the aspects of sharing knowledge and information demand. [26] and [27] considers the reputation of authors and its impact on the quality of articles in online communities. We extend their approach regarding the calculation of the reputation of an author by considering the demand for his articles. Our approach exploits the interactive character of Web 2.0 technologies. An analysis of information quality in corporate wikis with the help of five criteria is presented by [15]. It conducts surveys to assess the quality of a corporate wiki. In contrast to that approach, we calculate KPIs for that purpose. [28] presents a methodology for construction and validation of information quality metrics for Wikipedia. The methodology is comparable to our work, however they don't consider the aspect of information demand and don't provide any measures for using the metrics to improve information quality. [29] proposes measures for quantifying the success of knowledge management, which is comparable to our work. However, the methodologies are different. While [29] conducts surveys, we calculate KPIs for assessing the success of knowledge management.

\section{Conclusion and Outlook}

We discussed the potential of knowledge management for companies supported by Web 2.0 technologies and addressed the efficiency of this approach. This can be examined by focusing on the information quality, which in turn, needs further quantification. For this purpose, we presented a methodology for assessing and improving the quality of information provided by corporate wikis. Regarding the assessment, we presented two KPIs, i.e., relative demand and relative usefulness, including the corresponding process and data model. Regarding improving the quality, we used the KPIs to classify the articles. For this classification we introduced four categories and discussed possible actions for reducing information overload and increasing the visibility of articles. We analyzed the efficiency of our methodology with the help of an existing corporate wiki.

Based on the results of this paper, we intend to broaden our methodology to define additional KPIs for assessing the objective information need. For this, we analyze the combination of workflow systems supporting processes and corporate wikis providing help for the appropriate usage of those workflow systems. We study the context of process flows and their activities and information demands to identify the objective information need. In other words, we analyze whether an article is useful from the perspective of an employee interacting with a workflow system. In addition, we extend our work by considering further aspects of searching information in a corporate wiki to better understand the information demand.

\section{References}

[1] S. Güldenberg, Wissensmanagement und Wissenscontrolling in lernenden Organisationen. Deutscher Universitätsverlag, 2003.

[2] C. W. Holsapple and K. D. Joshi, "An investigation of factors that influence the management of knowledge in organizations," Journal of Strategic Information Systems, vol. 9, pp. 235-261, 2000.

[3] E. M. Noam, "Visions of the Media Age: Taming the Information Monster," in Multimedia A Revolutionary Challenge Prosepects for the Information Society Third Annual Colloquium, 1995.

[4] K. North and G. Kumta, Knowledge Management Value Creation Through Organizational Learning. Springer International Publishing, 2014.

[5] D. K. Yoo, M. A. Vonderembse, and T. Ragu-Nathan, "Knowledge quality: antecedents and consequence in project teams," Journal of Knowledge Management, vol. 15, no. 2, pp. 329-343, 2011.

[6] K. Kirchner, L. Razmerita, and F. Sudzina, "New Forms of Interaction and Knowledge Sharing on Web 2.0," in Web 2.0, M. D. Lytras, E. Damiani, and P. O. de Pablos, Eds. Springer, 2009.

[7] M. J. Eppler and J. Mengis, "The Concept of Information Overload: A Review of Literature from Organization Science, Accounting, Marketing, MIS, and Related Disciplines." Information Society, vol. 20, no. 5, pp. 325 - 344, 2004.

[8] D. Parmenter, Key Performance Indicators: Developing, Implementing, and Using Winning KPIs, 3rd ed. Wiley, 2015.

[9] T. O'Reilly, "What is web 2.0 design patterns and business models for the next generation of software," O'Reilly Network, 2005.

[10] I. Nonaka and H. Takeuchi, The Knowledge-creating Company: How Japanese Companies Create the Dynamics of Innovation. Oxford University Press, 1995.

[11] I. Nonaka and R. Toyama, "The knowledge-creating theory revisited: Knowledge creation as a synthesizing process," Knowledge Management Resource \& Practice, 2003.

[12] K. Stein and S. Blaschke, "Corporate wikis: A comparative analysis of structures and dynamics," in Fifth Conference Professional Knowledge Management: Experiences and Visions, March 25-27, 2009 in Solothurn, Switzerland, 2009, pp. 77-86.

[13] I. Lykourentzou, F. Dagka, K. Papadaki, G. Lepouras, and C. Vassilakis, "Wikis in enterprise settings: a survey," Enterprise Information Systems, vol. 6, no. 1, pp. 1-53, 2012.

[14] G. Kane, "A multimethod study of information quality in wiki collaboration," ACM Transactions on Management Information Systems 2, 2011.

[15] T. Friberg and W. Reinhardt, "An Empirical Study on Criteria for Assessing Information Quality in Corporate Wikis," in 14th International Conference on Information Quality, 2009.

[16] M. J. Eppler, Managing Information Quality. Springer, 2006.

[17] D. Strong, Y. Lee, and R. Wang, "Data quality in context," Соттиnications of the ACM Vol 40 No. 5, 1997.

[18] A. Picot and E. Franck, "Die Planung der Unternehmensressource Information (II)," wiso das wirtschaftsstudium, vol. 11, pp. 603-608, 1988.

[19] K. Balog, Y. Fang, M. de Rijke, P. Serdyukov, and L. Si, "Expertise Retrieval," Foundations and Trends in Information Retrieval, vol. 6, no. 2-3, pp. 127-256, 2012.

[20] A. Lih, "Wikipedia as Participatory Journalism: Reliable Sources? Metrics for evaluating collaborative media as a news resource," in 5th International Symposium on Online Journalism, 2004. 
[21] D. Wilkinson and B. Huberman, "Cooperation and quality in Wikipedia,' WikiSym '07 - Proceedings of the 2007 international symposium on Wikis, 2007.

[22] Object Management Group - (OMG), Business Process Model and Notation (BPMN), Std., 2011, http://www.omg.org/spec/BPMN/2.0/.

[23] P. Harmon and C. Wolf, "State of Business Process Management 2016," BPTrends, Tech. Rep., 2016.

[24] B. Henderson. (1974) The Experience Curve Reviewed. [Online]. Available: https://www.bcgperspectives.com/content/ Classics/strategy_supply_chain_management_experience_curve_ reviewed_the_concept/cc

[25] J. E. Blumenstock, "Size Matters: Word Count As a Measure of Quality on Wikipedia," in Proceedings of the 17th International Conference on World Wide Web. New York, NY, USA: ACM, 2008, pp. 1095-1096.

[26] B. T. Adler and L. de Alfaro, "A content-driven reputation system for the wikipedia," in Proceedings of the 16th International Conference on World Wide Web, WWW 2007, Banff, Alberta, Canada, May 8-12, 2007, 2007, pp. 261-270

[27] E.-P. Lim, B.-Q. Vuong, H. W. Lauw, and A. Sun, "Measuring qualities of articles contributed by online communities," in Web Intelligence, 2006. WI 2006. IEEE/WIC/ACM International Conference on, Dec 2006, pp. 81-87.

[28] B. Stvilia, L. Gasser, M. B. Twidale, and L. Smith, "A framework for information quality assessment," Journal of the American Society for Information Science and Technology, vol. 58, no. 12, pp. 1720-1733, 2007.

[29] M. E. Jennex, S. Smolnik, and D. Croasdell, "The search for knowledge management success," in 2016 49th Hawaii International Conference on System Sciences (HICSS), Jan 2016, pp. 4202-4211. 